\title{
In-Situ TEM Observations of Colloidal Particles in Liquids
}

\author{
H. Zheng,* A.M. Minor,* A.P. Alivisatos** and U. Dahmen* \\ * National Center for Electron Microscopy, Materials Sciences Division, LBNL, Berkeley, CA \\ 94720 \\ ** Materials Sciences Division, LBNL, Berkeley, Ca 94720
}

One of the most challenging tasks in electron microscopy is the observation of mechanisms and dynamics of processes in materials. The ability to adapt sample geometries to the microscale has made it possible to investigate fundamental features such as crystal growth, phase transformations or plastic deformation of materials in real time. This field of research has expanded tremendously over the past several years, largely because MEMS and AFM technology can now readily be adapted to electron microscope stages and samples.

In this work, we have used microlithography techniques to develop a self-contained liquid cell that can be used in any $3 \mathrm{~mm}$ TEM sample holder. The cell design is similar to that used by Williamson et al. to study cluster growth during electrodeposition of $\mathrm{Cu}$ [1]. As shown schematically in Figure 1, a thin layer of liquid is confined between two electron-transparent $\mathrm{SiN}$ windows approximately $25 \mathrm{~nm}$ thick. The liquid sample is connected to two reservoirs, which are filled with liquid solution prior to sealing the device and loading it into the electron microscope.

Using this cell, we have directly observed the trajectories of individual colloidal nanocrystals in solution [2]. Tracking real-time diffusion of both spherical $(5-15 \mathrm{~nm})$ and rod-shaped $(5 \times 10 \mathrm{~nm}) \mathrm{Au}$ nanocrystals in a thin film of water- $15 \%$ glycerol reveals trajectories that are more complex than previously thought. Figure 2 shows that the Au particles undergo complex movements, such as rolling coupled to large-step motions and macroscopic violations of the Stokes-Einstein relation for diffusion. An analysis of the measured trajectory of an individual $5 \mathrm{~nm} \mathrm{Au}$ particle shows particle motion to be composed of swarms of small steps interspersed with jumps corresponding to peaks in the graph. As drying patches form during the final stages of evaporation, particle motion becomes dominated by the nearby retracting liquid front.

We have also investigated the growth of colloidal platinum nanocrystals in solution and observed that normal growth and coalescence can proceed at the same time and in the same area of the sample [3]. This is illustrated in Figure 3 with a few frames extracted from a video sequence of the growth process. Despite such unexpectedly complex growth trajectories it is found that the particles evolve toward a monodisperse distribution. This talk will present these and other observations of mechanisms and dynamics during nanoparticle growth in liquid solution [4].

\section{References}

[1] M. Williamson et al. Nature Materials 2 (2003) 532.

[2] H. Zheng et al. Nano Letters 9 (2009) 2460.

[3] H. Zheng et al. Science 324 (2009) 1309

[4] This work is supported by the Director, Office of Science, Office of Basic Energy Sciences, of the U.S. Department of Energy under Contract No. DE-ACO3-76SFOOO98. 


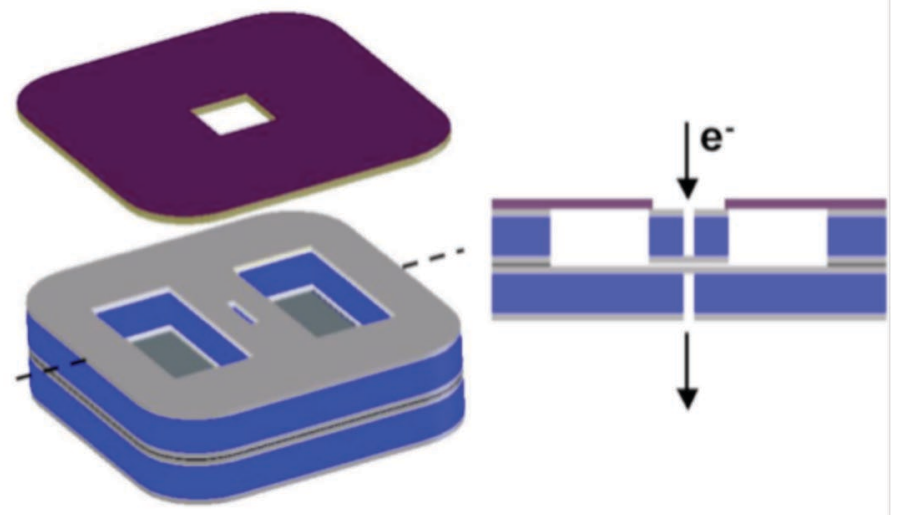

FIG. 1. Schematic of self-contained liquid cell in perspective and crosssectional view. Cell dimensions are $3 \mathrm{~mm}$ across the diagonal, with $0.6 \times 1.2 \times 0.1 \mathrm{~mm}$ reservoirs and $25 \mathrm{~nm}$ thick $1 \times 50 \mu \mathrm{m}$ electron transparent windows.
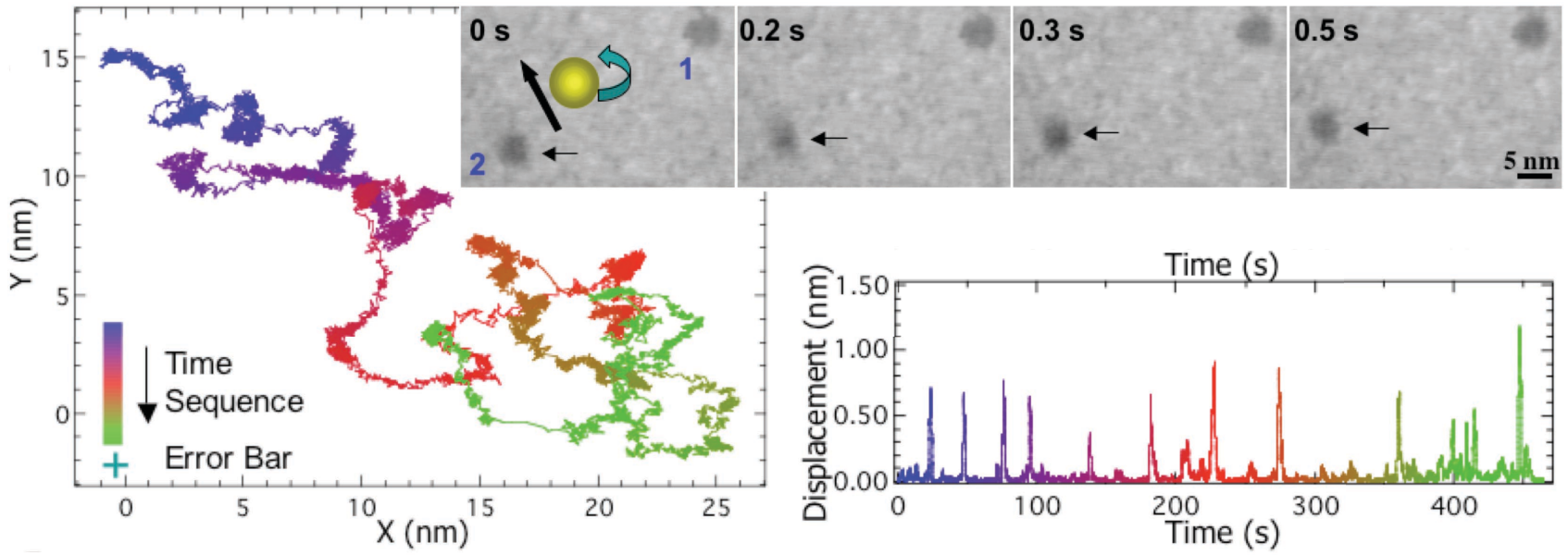

FIG. 2. Trajectory of a $5 \mathrm{~nm}$ particle's walk in a liquid thin film over a period of 500s, and a statistical analysis of its displacement (over 2 s intervals) versus time. Selected video frames showing the motion of two particles during a time interval of $0.5 \mathrm{~s}$. Particle 2 moved a large distance, with changes in crystal orientation indicated by changes in its diffraction contrast, while particle 1 shows little translation and no obvious change in its orientation [2].

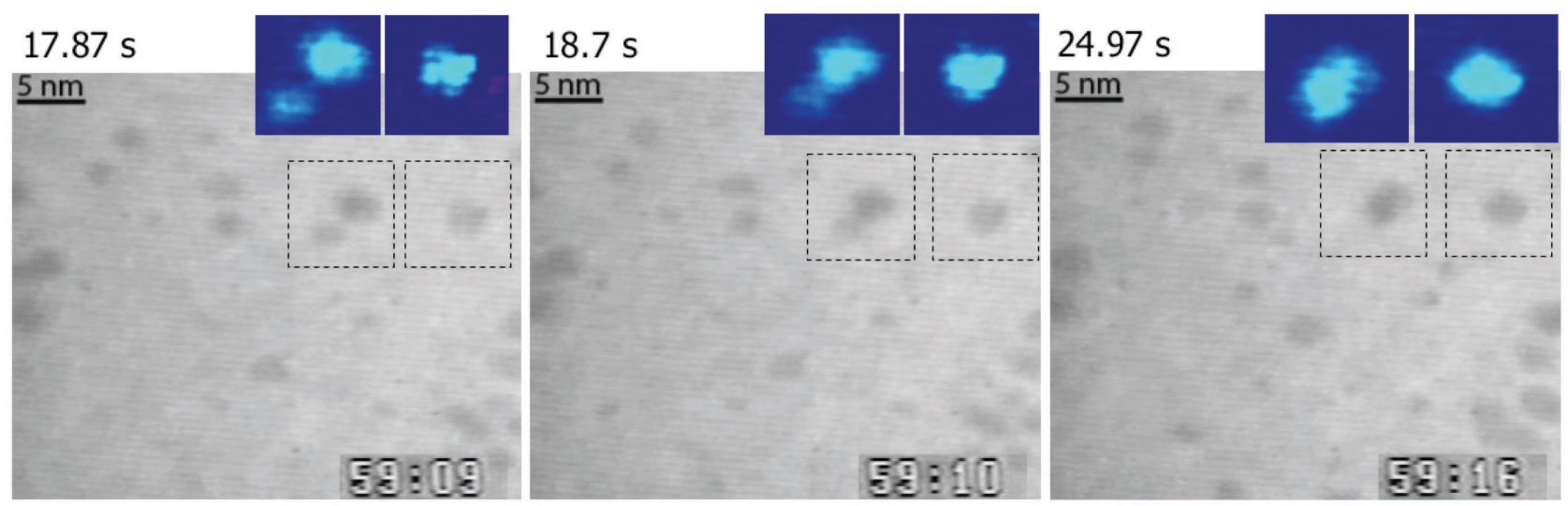

FIG. 3. Sequence from a video recording of Pt particles in liquid solution, showing coalescence and normal growth occurring in parallel [3]. 